\title{
Adaptación de dos medidas de apoyo social en estudiantes universitarios argentinos
}

\author{
Adaptation of two measures of social support in \\ argentinian university students
}

\author{
Mauricio Federico Zalazar Jaime ${ }^{1}$ \\ Marcos Cupani ${ }^{2}$ \\ Consejo Nacional de Investigaciones Científicas y Técnicas \\ Universidad Nacional de Córdoba, Argentina
}

Resumen. El soporte social constituye un importante constructo en el ámbito educativo, el cual influye en términos comportamentales, cognitivos y afectivos. Aunque en la literatura internacional existen numerosos instrumentos que permiten evaluar dicho constructo, en Argentina no se dispone de escalas que permitan evaluar el apoyo percibido por los estudiantes. Por lo tanto, el objetivo de este trabajo consistió en adaptar las escalas de Apoyo Social para Niños y Adolescentes (Malecki, Demaray \& Elliot, 2003) y Soporte Académico (Lent et al., 2005) en estudiantes universitarios. Para ello, en este estudio participaron 551 estudiantes de diferentes carreras dictadas en la Universidad Nacional de Córdoba (Argentina). Para ambos instrumentos, los resultados indicaron valores satisfactorios de consistencia interna (confiabilidad compuesta, $\rho>.70$ ) y, además, se logró replicar la estructura original de cada medida mediante análisis factorial confirmatorio. Se destacan las limitaciones y se sugieren nuevas líneas de estudio.

Palabras clave. Escala de apoyo social para niños y adolescentes, soporte académico, soporte social, estudiantes universitarios.

Abstract. Social support is an important construct in education which influences behavioral, cognitive and affective terms. Although there are numerous instruments to assess this construct, in Argentina there are no published scales to assess perceived support by students. Therefore, our aim was to adapt Social Support for Children and Adolescents (Malecki, Demaray \& Elliot, 2003) and Academic Support (Lent et al., 2005) scales in college students. For this projectIn this regard, data from 551 students of different degrees from the National University of Cordoba (Argentina) was collected. For both instruments, results showed satisfactory internal consistency values (composite reliability, $\rho>.70$ ) and the original structure was replicated for each measure using confirmatory factor analysis. Limitations and new lines of research are suggested.

Key words. Social support scale for children and adolescents, academic support, social support, university students.

\footnotetext{
${ }^{1}$ Mauricio Federico Zalazar - Jaime. CIPSI, Grupo Vinculado CIECS-CONICET-UNC. Facultad de Psicología, Universidad Nacional de Córdoba. Dirección Postal: Enfermera Gordillo esquina Enrique Barros, Ciudad Universitaria. Córdoba, Argentina, Estafeta Postal 32. Córdoba (5000), República Argentina. Email: mfzalazar@gmail.com

${ }^{2}$ Marcos Cupani. Laboratorio de Psicología de la Personalidad -CIPSI Grupo Vinculado CIECS-UNC-CONICET. Facultad de Psicología, Universidad Nacional de Cordoba,Argentina. Email: marcoscup@gmail.com
}

\section{@ $\odot \Theta \Theta$}

Esta obra está bajo una licencia de Creative Commons Reconocimiento-NoComercial-SinObraDerivada 4.0 Internacional. 


\section{Introducción}

Uno de los tópicos más frecuentes en la literatura educacional ha estado a cargo de identificar aquellas variables que contribuyen a predecir el rendimiento y la persistencia académica (Smith et al., 2003; Restubog, Florentino, \& Garcia, 2010). Sin embargo, gran parte de los estudios se han centrado en aspectos cognitivos, dejando de lado importantes variables ambientales (e.g. sociales y culturales-contextuales), las cuales suponen influencias en el sujeto y en otros aspectos del comportamiento de la carrera (Lent, Brown, \& Hackett, 2000). En este punto, la percepción de soporte social ha reportado contribuciones mediante una adecuada adaptación escolar (Cook, Herman, Phillips, \& Settersten, 2002), disminución de problemas de comportamiento (Domitrovich \& Bierman, 2001) y manejo de los problemas emocionales (Reddy, Rhodes, $\&$ Mulhall, 2003).

Una amplia variedad de definiciones ha sido elaborada respecto al soporte social (e.g. Cobb 1976; House, 1981), destacándose la clasificación desarrollada por Tardy (1985). Este autor distingue cinco dimensiones del soporte social; a saber, dirección, disposición, descripción/evaluación, contenido y red. La primera refiere a si se está recibiendo, o no, el apoyo social; la segunda, describe la disponibilidad en cuanto al acceso a determinado soporte y los recursos que puede propiciar el mismo. Por otro lado, el apoyo social implica indagar qué tipo de apoyo recibe una persona y el modo en cómo la percibe (descripción), mientras que la evaluación es la satisfacción que percibe el estudiante ante un soporte en particular. En cuanto al contenido, existen cuatro modalidades; apoyo emocional (tales como estima y empatía), instrumental (vinculado a conductas de ayuda), informativo (destinado a brindar consejos y/o asesoramiento) y evaluación (implica retroalimentación evaluativa). Por su parte, la red especifica al conjunto de personas que prestan su apoyo, como familiares, amigos, vecinos, compañeros de trabajo y profesionales de la comunidad, entre otros.

Uno de los instrumentos desarrollados bajo el modelo propuesto por Tardy (1985), es la escala de Apoyo Social para Niños y Adolescentes (CASSS por sus siglas en inglés; Malecki, Demaray, \& Elliot, 2003). Malecki et al. (2003) entienden al soporte social como la percepción de apoyo general o de conductas específicas, que proviene de personas de la red social del individuo, las cuales falicitan el afrontamiento de situaciones adversas que pueden incluir estrés (Malecki \& Demaray, 2002). A diferencia de otras medidas presentes en la literatura, esta escala brinda información respecto a la frecuencia e importancia en la percepción de apoyo proveniente de padres, profesores, compañeros de clase, amigos cercanos y personas de la escuela y, además, discrimina por cada fuente de soporte las cuatro modalidades de contenido antes mencionados. Diferentes estudios (Demaray \& Malecki, 2002a; Demaray \& Malecki, 2003a; Malecki \& Demaray, 2003) han reportado resultados de consistencia interna satisfactorios $(\alpha>$ .90) tanto para frecuencia como para importancia de cada soporte, y estudios de confiabilidad test-retest $(r$ comprendidos entre $.75-.78$ para frecuencia y $.45-.65$ para importancia; Malecki et al., 2003). En otro estudio conducido por Rueger, Malecki y Demaray (2010), los autores obtuvieron evidencia de estructura interna (mediante Anaisis Factorial Exploratorio) replicando la estructura original de cinco factores. También, las escalas del CASSS presentaron evidencia de validez convergente ( $r$ entre .49 y .62) con las escalas teóricamente semejantes de Social Skills Rating System (Gresham \& Elliott, 1990) y Student Self-Concept Scale (Gresham, Elliott, \& Evans-Fernandez, 1993) y validez discriminante $(r$ entre -.36 y -.41) con las sub-escalas de inadaptación clínica y síntomas emocionales pertenecientes a la Behavior Assessment Scale for Children Self Report of Personality (Reynolds \& Kamphaus, 1998).

Diferentes investigaciones (Elias \& Haynes, 2008; Skinner, Furrer, Marchand, \& Kindermann, 2008) han destacado contribuciones diferenciales según la fuente que propicie el soporte. Algunos estudios (Wettersten et al., 2005) han demostrado que el apoyo de los padres implica un mayor nivel de compromiso, participación y aspiración profesional. Otros trabajos (Malecki \& Demaray, 2003; Elias \& Haynes, 2008; Skinner et al., 2008) han evidenciado que el apoyo de los docentes está relacionado con un aumento en la autonomía, 
participación en el aula, menores problemas de comportamiento y un mayor rendimiento/persistencia académica. También se ha observado que el soporte recibido por los compañeros de clase y amigos cercanos se ha asociado con el apoyo emocional (Schultheiss, Palma, Predragovich \& Glasscock, 2002), aunque algunos estudios (Demaray \& Malecki, 2002a; 2002b) establecen que el apoyo propiciado por los compañeros de clase es más importante que el brindado por los amigos cercanos.

Otras investigaciones se han concentrado en inspeccionar las diferencias según el sexo de los estudiantes en la percepción del soporte social. Algunos estudios (Demaray \& Malecki 2002b; Malecki \& Demaray, 2003; Rueger, Malecki, \& Demaray, 2008) destacan que las mujeres se encuentran más atentas a los mensajes de apoyo de sus pares (compañeros de clase y amigos cercanos), mientras que los hombres perciben mayor apoyo de sus amigos cercanos. Por otro lado, Rueger et al. (2010) encontraron diferencias en cuanto a la percepción de padres y profesores, destacando que los varones son más propensos a percibir soporte de ambas fuentes. Del mismo modo, existe un volumen de trabajos (Demaray \& Malecki 2002b; Malecki \& Demaray, 2003; Rueger et al., 2008) que no han evidenciado diferencias de sexo con respecto al soporte percibido por los padres.

Otra medida presente en la literatura es la escala de Soporte Académico (Lent et al., 2005). Si bien este instrumento también indaga el apoyo percibido por los estudiantes respecto a los profesores, amigos y familiares, dicha escala representa una versión más breve que el CASSS y, además, no diferencia la fuente que propicia el soporte. Es decir, considera el apoyo percibido como una medida general. Lent et al. (2005) han destacado valores satisfactorios de consistencia interna $(\alpha>.80)$, evidencia de estructura interna (Análisis Factorial Confirmatorio) y estudio de validez convergente $(r=.41)$ con la escala de dominio de soporte social (Ezeofor \& Lent, 2014).

Por consiguiente, en este trabajo se ha propuesto traducir y adaptar las escalas de Apoyo Social para Niños y Adolescentes (CASSS) y Soporte Académico en una muestra de estudiantes pertenecientes a la Universidad Nacional de Córdoba (Argentina). De este modo, se cree brindar una contribución significativa al instrumental evaluativo empleado por los educadores y psicólogos. Además, en aquel contexto no se registran investigaciones que se ocupen de las relaciones entre las medidas de Apoyo y Soporte Social y la deserción académica, variable que adquiere relevancia en la educación pública universitaria donde se reportan altos porcentajes de abandono (alrededor del 59.6\%), producido, generalmente, en los primeros años de formación (Secretaría de Políticas Universitarias, 2013).

Entre las posibles causas de este fenómeno, puede destacarse que los estudiantes universitarios se enfrentan con una serie de circunstancias, tanto personales como contextuales, que difieren de sus experiencias educativas en los niveles previos (primario y secundario). Entre ellas, se destacan nuevos métodos de enseñanza, desproporción en la relación docentealumno, mayor presión de desempeño, desarrollo de estrategias autónomas de aprendizaje, adaptación a un nuevo contexto académico, abandono de su hogar, aislamiento afectivo y dificultades financieras (Verger et al., 2009). En este punto, el soporte social es una variable de importancia en el proceso de retención académica, e influye en términos comportamentales, cognitivos y afectivos (Schultheiss et al., 2002; Skinner et al., 2008). Por otro lado, algunos estudios (Miranda, Bilot, Peluso, Berman, \& Van Meek, 2006) han destacado que los estudiantes pertenecientes a otras minorías étnicas son más propensos a presentar ciertas dificultades en su proceso de adaptación al contexto educativo, destacando que, tanto la familia (Fuligni, Tseng, \& Lam, 1999) como el grupo de pares (Crockett et al., 2007), ejercen un rol protector. Si bien en Argentina no existe una diversificación de etnias como sucede en otros países, se destaca una prevalencia de ascendentes europeos; esta ha influenciado la cultura local principalmente en la zona centro del país. En adición a ello, la cantidad de estudiantes que asisten a la universidad provienen tanto de la ciudad de Córdoba (donde se encuentra dicha institución académica) como del interior provincial e interior del país. Con base en estos antecedentes, la procedencia podría 
llegar a mediar la relación entre los estudiantes y la fuente de soporte social. Particularmente, es posible pensar que los estudiantes del interior provincial y de otras provincias tienden a percibir un mayor nivel de soporte social a diferencia de los estudiantes oriundos de la ciudad de Córdoba. Del mismo modo, podría considerarse que el género de los estudiantes puede influir en la percepción del apoyo y soporte social (Demaray \& Malecki 2002b; Malecki \& Demaray, 2003; Rueger et al., 2008).

\section{Metodología}

\section{Participantes}

En este estudio, participaron 551 estudiantes de la Universidad Nacional de Córdoba (UNC, Argentina), de los cuales $404(73.3 \%)$ eran de sexo femenino y 125 $(22.7 \%)$ de sexo masculino, con edades comprendidas entre los 17 y 65 años $(M=21.97 ; D E=6.32)$. Del total de la muestra, 384 participantes se encontraban realizando el curso de ingreso a la facultad de Psicología (UNC), mientras que los restantes 167 alumnos se encontraban cursando otras carreras en la misma universidad distribuidas en las siguientes áreas Arte y Humanidades, 1.5\%; Tecnología, 2\%; Ciencias Naturales, $16 \%$; y Ciencias Sociales, $10.9 \%$.

\section{Instrumentos}

Escala de Apoyo Social para Niños y Adolescentes (CASSS; Malecki et al., 2003). La escala está compuesta por 60 ítems dividida en cinco sub-escalas de 12 ítems, donde cada uno evalúa la percepción que poseen los estudiantes acerca de sus padres, profesores, compañeros de clase, amigos cercanos y personas de la escuela. Cada participante debe responder a cada enunciado mediante dos tipos de respuesta a) frecuencia, evaluada con escala Likert de seis opciones de respuesta, desde 1 "nunca" a 6 "siempre", y b) importancia, con una escala Likert con tres opciones de respuesta, desde 1 "no es importante" a 3 "muy importante". En su versión original, este instrumento posee estudios de consistencia interna (coeficiente alfa de Cronbach), desde .90 a .95 , estabilidad temporal $(r$ entre .75 y .78) y evidencia de validez de estructura interna y convergente-discriminante.
Soporte Académico (Lent et al., 2005). Este instrumento está compuesto por nueve ítems que evalúan, de modo general, el apoyo recibido por los estudiantes de parte de profesores, amigos y familiares respecto a su carrera académica. Los participantes deben responder utilizando una escala tipo Lickert con cinco opciones de respuesta donde 1 representa "totalmente en desacuerdo" y 5 "totalmente de acuerdo". En un estudio realizado por Lent, Singley, Sheu, Schmidt y Schmidt (2007), los autores reportaron estudios de consistencia interna (alfa de Cronbach) de $\alpha=.84$.

\section{Traducción de los items}

Se realizó una traducción (método directo) de los items del CASSS y Soporte Académico. Ocho expertos en la lengua inglesa, cuatro por cada instrumento, tradujeron los items del idioma original (inglés) al español. Luego, las diferentes versiones fueron comparadas y se realizaron los ajustes idiomáticos pertinentes, procurando mantener cierta equivalencia conceptual, semántica y funcional más que una mera traducción literal (Mimura \& Griffiths, 2008). Particularmente, se inspeccionaron aquellas expresiones que no son habituales. Por ejemplo, la expresión "mentor" de un ítem de la escala de Soporte Académico ("have access to a mentor who could offer me advice and encouragement") se la sustituyó por otra palabra (referente) más adecuada a nuestro contexto y de uso cotidiano ("tengo acceso a un referente que me podría brindar consejos y apoyo"). Respecto a la sub-escala del CASSS denominada "personas de la escuela" se la redefinió como "personas de mi facultad".

\section{Procedimiento}

Los cuestionarios fueron administrados de manera colectiva, en horario regular de clases bajo la supervisión del primer autor de este trabajo. Previa a la administración, se brindó toda la información necesaria sobre el trabajo a desarrollar y los objetivos del mismo en un lenguaje claro y comprensible. Se destacó la libre voluntad de participar y que la información obtenida sería confidencial, utilizada solo para fines de investigación, resguardando en todo momento la identidad de los participantes. Además, se 
brindó a cada estudiante una nota de consentimiento informado donde los participantes dejaron constancia explícita sobre su interés en participar. Luego, se administraron los cuestionarios CASSS y Soporte Académico. La duración de la administración fue de 25 minutos. El tipo de muestreo fue no probabilístico del tipo accidental (Bologna, 2010).

\section{Estrategia de análisis}

Se utilizó el software SPSS para Windows versión 19.0 con el fin de preparar los datos para los análisis propuestos. Los análisis conducidos en este trabajo se realizaron tanto para la CASSS como para la escala de Soporte Académico. En primer lugar, se evaluó el patrón de los valores perdidos, con el fin de estimar si el mismo respondía a una distribución aleatoria (Tabachnick \& Fidell, 2011). Se calculó la media, desviación estándar, asimetría y curtosis. Como criterio para evaluar los índices de asimetría y curtosis, se consideraron como excelentes valores entre \pm 1.00 , y adecuados valores inferiores a \pm 2.00 (George \& Mallery, 2011). Se identificaron los casos atípicos univariados mediante el cálculo de puntuaciones $\mathrm{Z}$ para cada variable (puntuaciones $Z> \pm 3.29$ fueron considerados atípicos).

Con el fin de replicar las estructuras originales (cinco factores del CASSS; un factor de la escala de Soporte Académico), se condujo un Análisis Factorial Confirmatorio (AFC). Para ello, se utilizó el software estadístico Mplus versión 6.12, ya que permite aplicar el método de estimación denominado mínimos cuadrados ponderados (WLS), considerado el más oportuno cuando se trata con datos categóricos (escalas Likert, por ejemplo; Flora \& Curran, 2004). Para evaluar el ajuste de los modelos, se utilizaron los estadísticos Chi-Cuadrado, el Índice de Ajuste Comparativo (CFI), el Índice de Tucker-Lewis (TLI), el Error Cuadrático Medio de Aproximación (RMSEA) y la ponderada Media Cuadrática Residual (WRMR). Los valores entre .90 y .95 o superiores para el CFI y TLI son considerados como ajustes aceptables a excelentes, se esperan valores entre .05 y .08 para el RMSEA y valores menores a 1.00 para WRMR (Yu \& Muthén, 2002). Posteriormente, se estimó la confiabilidad compuesta $(\rho)$. Este método utiliza las cargas factoriales, varianza residual y $r^{2}$ de cada ítem según una estructura subyacente, presentando, de esta manera, una menor variación del error. Valores iguales o superiores a $\rho=.70$ son considerados como aceptables (Nunnally, 1978).

Se realizó un análisis de la varianza con el fin de inspeccionar si la percepción de soporte social difiere según la procedencia de los estudiantes. Previo a ello, se realizó una prueba $t$ diferencia de media para inspeccionar si existen diferencias según el sexo de los estudiantes. En ambos estudios se consideró el estadístico d de Cohen (prueba $t$ ) y el coeficiente eta cuadrado parcial ( $\eta^{2}$, ANOVA); para su interpretación, se consideraron valores pequeños $\left(d=|.2|, \eta^{2}=1 \%\right)$, medianos, $\left(d=|.5|, \eta^{2}=10 \%\right)$, y grandes $\left(d=|.8|, \eta^{2}\right.$ $=25 \%$; Cohen, 1988).

Para dotar de mayor claridad la presentación de los resultados, se organizaron en tres fases. En las primeras dos se reportan los resultados de los análisis propuestos previamente para la escala CASSS y Soporte Académico; mientras que, en la tercera se desarrolla el estudio de validez convergente entre ambos instrumentos con el fin de corroborar la equivalencia entre ambos constructos.

\section{Resultados}

Fase 1. Escala de Apoyo Social para Niñosy Adolescentes (CASSS).

Preparación de los datos y análisis de items. Los casos perdidos según la frecuencia de los soportes oscilaron entre $0.5 \%$ (e.g. ítem 1 , factor padres) a $4.4 \%$ (ítem 58 , factor personas de mi facultad); mientras que, según la importancia, variaron entre $3.5 \%$ (ítem 6 , factor padres) a $6.9 \%$ (item 55 , factor personas de mi facultad). Considerando que la dimensión importancia superó el 5\% establecido por la literatura (Schafer, 1999), se realizó la prueba de Little (1988) para determinar el patrón de casos perdidos. Este estudio indicó un patrón completamente al azar (MCAR; $\chi^{2}=4799,09, \mathrm{gl}=4874$, $p \geq .775)$. En virtud de estos resultados, se procedió a imputar los datos mediante el modo.

Respecto a la asimetría y curtosis, se observó que, según la frecuencia, 53 ítems presentaron valores entre 
\pm 1.00 , y 7 ítems presentaron valores entre \pm 2 . Según la importancia, 58 ítems presentaron valores entre \pm 1.00 y 2 ítems presentaron valores entre \pm 2 . En cuanto a los casos atípicos, se identificaron 32 casos atípicos univariados según la frecuencia de los soportes, y no se observaron casos atípicos según la importancia de los mismos. Considerando que la presencia de casos atípicos tiende a distorsionar los resultados, se retuvieron los mismos con el fin de evitar la limitación en la generalidad de los análisis ya que dejarían de representar a un segmento de la población en estudio (Hair, Anderson, Tatham, \& Black, 1999).

Análisis factorial confirmatorio (AFC). CASSS Frecuencia. Los resultados del AFC indicaron un ajuste adecuado a los datos (CFI .91, TLI .91, RMSEA .041 90\% CI .039 .043, WRMR 1.49). Los pesos de regresión estandarizados $(\phi \leq .05)$ en el factor padres oscilaron entre .42 a .74 ; profesores entre .56 a .75 ; compañeros de clase entre .59 a .73 ; amigos cercanos entre .64 a .76 , y en el factor personas de mi facultad entre .63 a .84. Considerando que el ajuste fue aceptable pero no óptimo según los criterios propuestos, se decidió inspeccionar los índices de modificación (IM). Estos indicaron una ganancia del ajuste al correlacionar los residuos entre los ítems 53 (me ayudan a resolver mis problemas dándome información) y 54 (me explican las cosas que no entiendo) y entre los ítems 55 (me dicen cuán bien me desempeño en las tareas) y 56 (me dicen que hice un buen trabajo cuando hago algo bien) ambos de la sub-escala personas de mi facultad. Se considera que cuando los residuos se encuentran altamente correlacionados es un indicador de redundancia debido a la superposición de contenidos (Brown, 2015). Por lo tanto, considerando que se trata de un solapamiento por la semejanza respecto al contenido en ambos pares de ítems, se decidió eliminar el que presentó un menor peso de regresión estandarizado (ítem 54 y 55). De este modo, el ajuste del modelo fue óptimo (CFI .95, TLI .94, RMSEA .042, 90\% CI .039 .044, WRMR 1.39). En la Tabla 1 se presentan las cargas factoriales de los ítems del CASSS.

Consistencia interna. Los valores obtenidos fueron satisfactorios y estuvieron comprendidos entre $\rho$
$=.92$ (padres) y $\rho=.95$ (personas de mi facultad). Adicionalmente, se estimó una medida general de soporte, sin discriminar la fuente de soporte, la cual también reportó resultados satisfactorios ( $\rho=.99$; ver Tabla 3 ).

Estudio de diferencia de grupos. Los resultados indicaron que las mujeres presentaron mayores niveles de apoyo en las escalas de soporte de padres $\left(M_{\text {masculino }}=46.18\right.$, $\left.M_{\text {femenino }}=48.63 ; t(235,10)=2.16, p .03, d=.21\right) \mathrm{y}$ amigos cercanos $\left(M_{\text {masculino }}=54.16, M_{\text {femenino }}=58.65\right.$; $t(180,17)=3.97, p .00, d=.43)$ a diferencia de los varones. Considerando los criterios establecidos por Cohen (1988) se observaron efectos pequeños (ver Tabla 3). Previo a la realización del ANOVA se descartaron 21 casos debido a que no presentaron respuesta alguna sobre su procedencia (16 casos), y en menor magnitud manifestaron procedencia de países extranjeros (cinco casos). Se observaron diferencias en las sub-escalas padres $\left[F(2,527)=12,38, p=.00 ; \eta^{2}\right.$ $=.13]$ tamaño del efecto grande; profesores $[F(2,527)$ $\left.=6,94, p=.00 ; \eta^{2}=.14\right]$ tamaño del efecto grande; $\mathrm{y}$ personas de mi facultad $\left[F(2,527)=11.19, p=.00 ; \eta^{2}\right.$ $=.09]$ tamaño del efecto mediano.

La comparación post hoc mediante la prueba HSD de Tukey indicó que aquellos estudiantes provenientes de otras provincias $(M=50.69, D E=10.88)$ como del interior provincial $(M=50.00, D E=10.43)$ tienden a percibir con mayor frecuencia el apoyo de sus padres, en comparación con los estudiantes propios de Córdoba $(M=45.37, D E=12.73)$. Por otro lado, se observaron diferencias respecto a la percepción de apoyo de los profesores, que fue mayor en estudiantes provenientes del interior provincial $(M=51.67, D E=$ 10.57) respecto a los estudiantes propios de la ciudad de Córdoba $(M=46.89, D E=11.98)$. Por su parte, en cuanto a las personas de mi facultad, los estudiantes provenientes del interior provincial $(M=41.25, D E=$ 13.14) presentaron una mayor percepción de soporte de esta fuente en comparación con los estudiantes de otras provincias $(M=35.33, D E=13.69)$ y de la ciudad de Córdoba $(M=34.39, D E=12.92$, ver Tabla 4$)$.

CASSS - Importancia. Los resultados del AFC (CFI .91, TLI .91, RMSEA .041 90\% CI .039.043, WRMR 1.49) indicaron un ajuste aceptable a los datos. Los pesos de 
regresión estandarizados $(\phi \leq .05)$ en el factor padres oscilaron entre .42 a .74 ; profesores entre .56 a .75 ; compañeros de clase entre .59 a .73 ; amigos cercanos entre .64 a .76 ; y personas de mi facultad entre .63 a .84 . Como se destacó previamente, a pesar de obtener un buen ajuste los índices de modificación indicaron un solapamiento entre los mismos pares de ítems enunciados anteriormente. Siguiendo los mismos criterios se eliminaron los ítems 53 y 56 . De este modo, el ajuste del modelo mejoró levemente (CFI .93, TLI .92, RMSEA .038, 90\% CI .035 .040, WRMR 1.37). En la Tabla 1 se presenta las cargas factoriales de los ítems del CASSS.

Consistencia interna. Los valores hallados fueron satisfactorios y oscilaron entre $\rho=.89$ (padres) y $\rho=$ .94 (personas de mi facultad; ver Tabla 3).

Estudio de diferencia de grupos. Los resultados indicaron que las mujeres, a diferencia de los varones, presentaron mayores niveles de apoyo en las escalas de padres $\left(M_{\text {masculino }}=24.50, M_{\text {femenenino }}=26.37 ; t(235,10)\right.$ $=4.06, p=.00, d=.41) \mathrm{y}$ amigos cercanos $\left(M_{m}\right.$ $=27.24, M_{\text {femenino }}=29.52 ; t(235,10)=4.90, p .00, d$ $=.49$ ). Adicionalmente, cuando se consideró una medida general de la importancia del soporte social, se observó que las mujeres tienden a considerar como más importante dicha percepción a diferencia de los varones $\left(M_{\text {masculino }}=124.60, M_{\text {femenino }}=130.12 ; t(214,76)\right.$ $=3.14, p=.00, d=.33)$. Considerando los criterios establecidos por Cohen (1988), se observaron efectos pequeños (ver Tabla 3). Los resultados del ANOVA indicaron diferencias en la sub-escala compañeros de clase $\left[\mathrm{F}(2,527)=4,59, p=.01 ; \eta^{2}=.07\right]$ tamaño del efecto mediano. La comparación post hoc mediante la prueba HSD de Tukey indicó que aquellos estudiantes provenientes del interior provincial $(M=25.42, D E$ = 4.89) como de Córdoba capital $(M=25.15, D E=$ 4.56) otorgan mayor importancia al soporte propiciado por los compañeros de clase en comparación con los estudiantes de otras provincias $(M=23.93, D E=4.82$, ver Tabla 4).

Fase 2. Soporte Académico

Preparación de los datos y análisis de items. Los casos perdidos variaron entre $0.2 \%$ (ítem 9) a 1.5\% (item
Tabla 1

Cargas factoriales del CASSS, según frecuencia e importancia

\begin{tabular}{|c|c|c|}
\hline Fuente & Frecuencia & Importancia \\
\hline \multicolumn{3}{|l|}{ Padres } \\
\hline CASSS_1 & .70 & .58 \\
\hline CASSS_2 & .67 & .63 \\
\hline CASSS_3 & .75 & .68 \\
\hline CASSS_4 & .78 & .67 \\
\hline CASSS_5 & .78 & .64 \\
\hline CASSS_6 & .76 & .67 \\
\hline CASSS_7 & .76 & .74 \\
\hline CASSS_8 & .66 & .67 \\
\hline CASSS_9 & .58 & .43 \\
\hline CASSS_10 & .61 & .64 \\
\hline CASSS_11 & .80 & .71 \\
\hline CASSS_12 & .58 & .42 \\
\hline \multicolumn{3}{|l|}{ Profesores } \\
\hline CASSS_13 & .72 & .65 \\
\hline CASSS_14 & .72 & .62 \\
\hline CASSS_15 & .72 & .56 \\
\hline CASSS_16 & .77 & .64 \\
\hline CASSS_17 & .73 & .64 \\
\hline CASSS_18 & .71 & .62 \\
\hline CASSS_19 & .66 & .66 \\
\hline CASSS_20 & .72 & .73 \\
\hline CASSS_21 & .70 & .68 \\
\hline CASSS_22 & .72 & .61 \\
\hline CASSS_23 & .78 & .70 \\
\hline CASSS_24 & .77 & .75 \\
\hline \multicolumn{3}{|c|}{ Compañeros de clase } \\
\hline CASSS_25 & .67 & .64 \\
\hline CASSS_26 & .54 & .67 \\
\hline CASSS_27 & .74 & .71 \\
\hline CASSS_28 & .73 & .61 \\
\hline CASSS_29 & .75 & .63 \\
\hline CASSS_30 & .77 & .73 \\
\hline CASSS_31 & .70 & .67 \\
\hline CASSS_32 & .67 & .72 \\
\hline CASSS_33 & .64 & .59 \\
\hline CASSS_34 & .77 & .71 \\
\hline CASSS_35 & .75 & .71 \\
\hline CASSS_36 & .81 & .71 \\
\hline
\end{tabular}

Continúa... 


\begin{tabular}{|c|c|c|}
\hline Fuente & Frecuencia & Importancia \\
\hline \multicolumn{3}{|c|}{ Amigos cercanos } \\
\hline CASSS_37 & .68 & .68 \\
\hline CASSS_38 & .77 & .69 \\
\hline CASSS_39 & .84 & .76 \\
\hline CASSS_40 & .82 & .76 \\
\hline CASSS_41 & .72 & .71 \\
\hline CASSS_42 & .70 & .74 \\
\hline CASSS_43 & .67 & .64 \\
\hline CASSS_44 & .63 & .75 \\
\hline CASSS_45 & .67 & .71 \\
\hline CASSS_46 & .84 & .70 \\
\hline CASSS_47 & .76 & .67 \\
\hline CASSS_48 & .82 & .75 \\
\hline \multicolumn{3}{|c|}{ Personas de mi facultad } \\
\hline CASSS_49 & .80 & .75 \\
\hline CASSS_50 & .84 & .81 \\
\hline CASSS_51 & .75 & .69 \\
\hline CASSS_52 & .79 & .72 \\
\hline CASSS_53 & .83 & .79 \\
\hline CASSS_54 & .83 & .81 \\
\hline CASSS_55 & .87 & .84 \\
\hline CASSS_56 & .87 & .81 \\
\hline CASSS_57 & .68 & .72 \\
\hline CASSS_58 & .79 & .67 \\
\hline CASSS_59 & .78 & .72 \\
\hline CASSS_60 & .70 & .63 \\
\hline
\end{tabular}

3). En virtud de estos resultados se procedió a imputar los datos mediante el modo. Respecto a la asimetría y curtosis, se observó que ocho ítems presentaron valores entre \pm 1.00 , y solo un ítem presentó un valor comprendido entre \pm 2 (George \& Mallery, 2011). Solo se reportaron 11 casos atípicos univariados los cuales se decidieron mantener.

Análisis factorial confirmatorio. Los resultados del AFC (CFI.85, TLI.80, RMSEA .1490\% CI.126.156, WRMR 1.71) indicaron un pobre ajuste a los datos. Los pesos de regresión estandarizados $(p \leq .05)$ oscilaron entre
.32 a .81. Una inspección de los índices de modificación permitió observar que un cambio en los parámetros estimados entre los errores pertenecientes al par de ítem 4 (recibo apoyo y asesoramiento de mi profesor, si siento que necesito ese tipo de ayuda) e item 6 (recibo asesoramiento valioso de mi profesor) produce una ganancia en el modelo. A diferencia del criterio anterior y con el objetivo de mantener los ítems originales de este instrumento, se decidió correlacionar los residuos. De este modo, el ajuste mejoró satisfactoriamente (CFI .97, TLI .95, RMSEA .07, 90\% CI .054 .087, WRMR .88). En la Tabla 2 se presentan las cargas factoriales de los ítems de la escala de Soporte Académico.

Consistencia interna. Se reportó un valor satisfactorio $(\rho=.76)$ para la escala de Soporte Académico.

Estudio de diferencia de grupos. Se reportaron diferencias $\left[\mathrm{F}(2,456)=4.33, p=.01 ; \eta^{2}=.06\right]$ entre aquellos estudiantes provenientes del interior provincial y los estudiantes propios de Córdoba, destacando un tamaño del efecto mediano. La comparación post hoc mediante la prueba HSD de Tukey indicó que aquellos estudiantes provenientes del interior provincial $(M=35.30, D E=$ 4.59) tienden a percibir mayor soporte que sus pares de Córdoba $(M=33.71, D E=4.32$, ver Tabla 4$)$.

Fase 3. Estudio de valider, convergente

Se obtuvo la matriz de correlaciones entre las diez sub-escalas del CASSS y la escala de Soporte Académico.

Tabla 2

Cargas factoriales de la escala de Soporte Académico

\begin{tabular}{cc}
\hline Soporte Académico & Carga factorial \\
\hline Ítem 1 & .51 \\
Ítem 2 & .73 \\
Ítem 3 & .47 \\
Ítem 4 & .54 \\
Ítem 5 & .57 \\
Ítem 6 & .59 \\
Ítem 7 & .38 \\
Ítem 8 & .29 \\
Ítem 9 & .46 \\
\hline
\end{tabular}


Cuando se consideró la frecuencia de las fuentes de soporte social, se observaron correlaciones moderadas a altas $\left(r_{\text {mediana }}=.36\right)$. En menor magnitud, la importancia de los soportes presentó correlaciones moderadas $\left(r_{\text {mediana }}=.22\right)$ con el Soporte Académico. De manera similar, cuando se consideraron las puntuaciones totales del CASSS, se observaron correlaciones moderadas con la escala de Soporte Académico $\left(r_{\text {frecuencia }}=.49, r_{\text {importania }}\right.$ $=.30$, ver Tabla 5), resultados que evidencian una convergencia moderada entre ambos constructos.

\section{Discusión}

El objetivo de este trabajo consistió en adaptar dos medidas de apoyo social. La primera, desarrollada por Malecki et al. (2003) la cual discrimina la fuente de percepción de soportes (padres, profesores, amigos cercanos, compañeros de clase y personas de la facultad) que pueden percibir los jóvenes y, por otro lado, la escala desarrollada por Lent et al. (2005) que considera al soporte como un constructo general, es decir, sin diferenciar la fuente que proporciona el soporte.

Respecto al CASSS, los resultados del estudio de estructura interna (análisis factorial confirmatorio) y confiabilidad fueron satisfactorios para las diferentes fuentes de soporte según su frecuencia e importancia. Sin embargo, a pesar de estos resultados, cabe destacar que la fuente personas de mi facultad presentó ciertas dificultades en su interpretación. Esto responde a que la escala desarrollada originalmente por los autores fue elaborada para evaluar la percepción de soporte en ámbitos educativos de nivel inicial y secundario. Al realizar el proceso de adaptación y cambiar el nombre

Tabla 3

Confiabilidad compuesta ( $\rho$ ), Media (M), Desviación estándar (DE), prueba t diferencias de medias y tamaño del efecto (d), de las medidas CASSS y Soporte Académico.

\begin{tabular}{|c|c|c|c|c|c|c|c|c|c|}
\hline \multirow[t]{2}{*}{ Instrumento } & & \multirow{2}{*}{$\rho$} & \multicolumn{2}{|c|}{ Masculino } & \multicolumn{2}{|c|}{ Femenino } & \multirow[b]{2}{*}{$t$} & \multirow[b]{2}{*}{$p$} & \multirow[b]{2}{*}{$d$} \\
\hline & & & $M$ & $D E$ & $M$ & $D E$ & & & \\
\hline \multicolumn{10}{|l|}{ CASSS } \\
\hline \multirow{4}{*}{ Frecuencia } & Padres & .92 & 46.18 & 10.69 & 48.63 & 12.31 & 2.16 & .03 & .21 \\
\hline & Profesores & .93 & 47.71 & 11.59 & 48.59 & 11.78 & 0.74 & .46 & .07 \\
\hline & Compañeros de clase & .93 & 50.26 & 10.05 & 50.18 & 11.29 & 0.07 & .94 & .01 \\
\hline & Amigos cercanos & .94 & 54.16 & 11.48 & 58.65 & 9.52 & 3.97 & .00 & .43 \\
\hline \multirow{8}{*}{ Importancia } & Personas de mi facultad & .95 & 36.54 & 13.40 & 36.13 & 13.62 & 0.30 & .77 & .03 \\
\hline & Total & 99 & 234.86 & 39.52 & 242.19 & 41.31 & 1.75 & .08 & .18 \\
\hline & Padres & .89 & 24.50 & 4.54 & 26.37 & 4.49 & 4.06 & .00 & .41 \\
\hline & Profesores & .90 & 26.49 & 4.60 & 27.16 & 4.50 & 1.45 & .15 & .15 \\
\hline & Compañeros de clase & .91 & 24.55 & 4.59 & 24.89 & 4.85 & 0.69 & .49 & .07 \\
\hline & Amigos cercanos & .93 & 27.24 & 4.96 & 29.52 & 4.41 & 4.90 & .00 & .49 \\
\hline & Personas de mi facultad & .94 & 21.82 & 5.37 & 22.19 & 5.21 & 0.68 & .50 & .07 \\
\hline & Total & .98 & 124.60 & 16.56 & 130.12 & 17.34 & 3.14 & .00 & .33 \\
\hline Soporte académico & & .76 & 33.90 & 4.78 & 34.41 & 4.65 & 0.97 & .34 & .11 \\
\hline
\end{tabular}


Tabla 4

Análisis de la varianza (ANOVA), Media (M), Desviación estándar (DE), y tamaño del efecto ( $\eta 2)$, de las subescalas del CASSS y la escala de Soporte Académico según la procedencia de los estudiantes

\begin{tabular}{|c|c|c|c|c|c|c|c|c|c|}
\hline \multirow[b]{2}{*}{ CASSS - Frecuencia } & \multicolumn{2}{|c|}{ Ciudad Córdoba } & \multicolumn{2}{|c|}{ Interior provincial } & \multicolumn{2}{|c|}{ Otras provincias } & \multirow[b]{2}{*}{$F$} & \multirow[b]{2}{*}{$p$} & \multirow[b]{2}{*}{$\eta^{2}$} \\
\hline & $M$ & $D E$ & $M$ & $D E$ & $M$ & $D E$ & & & \\
\hline Padres & 45.37 & 12.73 & 50.00 & 10.43 & 50.69 & 10.88 & 12,38 & .00 & .13 \\
\hline Profesores & 46.89 & 11.98 & 51.67 & 10.57 & 47.88 & 11.55 & 6,94 & .00 & .14 \\
\hline Compañeros de clase & 50.33 & 10.75 & 51.50 & 10.91 & 49.21 & 11.38 & 1,54 & .22 & .08 \\
\hline Amigos cercanos & 57.00 & 10.48 & 59.10 & 8.76 & 57.63 & 10.25 & 1,73 & .18 & .08 \\
\hline Personas de mi facultad & 34.39 & 12.92 & 41.25 & 13.14 & 35.33 & 13.69 & 11,19 & .00 & .09 \\
\hline \multicolumn{10}{|l|}{ CASSS - Importancia } \\
\hline Padres & 25.76 & 4.57 & 26.42 & 4.34 & 25.55 & 4.67 & 1,34 & .26 & .04 \\
\hline Profesores & 27.35 & 4.38 & 26.81 & 4.59 & 26.43 & 4.61 & 2,14 & .12 & .06 \\
\hline Compañeros de clase & 25.15 & 4.56 & 25.42 & 4.89 & 23.93 & 4.82 & 4,59 & .01 & .07 \\
\hline Amigos cercanos & 28.82 & 4.74 & 29.28 & 4.49 & 28.85 & 4.63 & 0,43 & .65 & .05 \\
\hline Personas de mi facultad & 21.85 & 4.73 & 22.69 & 5.17 & 21.92 & 5.92 & 1,08 & .34 & .08 \\
\hline Soporte Académico & 33.71 & 4.32 & 35.30 & 4.59 & 34.22 & 5.03 & 4,33 & .01 & .06 \\
\hline
\end{tabular}

Tabla 5

Matriz de correlaciones entre el CASSS y Soporte Académico

\begin{tabular}{|c|c|c|c|c|c|c|c|c|c|c|c|c|c|}
\hline & 1 & 2 & 3 & 4 & 5 & 6 & 7 & 8 & 9 & 10 & 11 & 12 & 13 \\
\hline 1. Padres $(F)$ & 1.00 & $.29 * *$ & $.34 * *$ & $.37 * *$ & $.27 * *$ & $.44^{* *}$ & $.15^{* *}$ & $.17 * *$ & $.20^{* *}$ & $.17 * *$ & $.65^{* *}$ & $.30 * *$ & $.36 * *$ \\
\hline 2. Profesores $(\mathrm{F})$ & & 1.00 & $.37 * *$ & $.31^{* *}$ & $.60 * *$ & $.16^{* *}$ & $.29 * *$ & $.11^{*}$ & $.13^{* *}$ & $.27 * *$ & $.75^{* *}$ & $.26^{* *}$ & $.43^{* *}$ \\
\hline 3. Compañeros de clase $(\mathrm{F})$ & & & 1.00 & $.40^{* *}$ & $.41 * *$ & $.14^{* *}$ & $.22 * *$ & $.54 * *$ & $.19^{* *}$ & $.23^{* *}$ & $.71 * *$ & $.36 * *$ & $.27 * *$ \\
\hline 4. Amigos cercanos $(\mathrm{F})$ & & & & 1.00 & $.23^{* *}$ & $.25^{* *}$ & $.18^{* *}$ & $.23^{* *}$ & $.52^{* *}$ & $.14^{* *}$ & $.63^{* *}$ & $.36^{* *}$ & $.29 * *$ \\
\hline 5. Personas de mi facultad $(\mathrm{F})$ & & & & & 1.00 & $.14^{* *}$ & $.22^{* *}$ & $.23^{* *}$ & $.16^{* *}$ & $.54^{* *}$ & $.75^{* *}$ & $.36^{* *}$ & $.36^{* *}$ \\
\hline 6. Padres (I) & & & & & & 1.00 & $.46^{* *}$ & $.41 * *$ & $.48^{* *}$ & $.26^{* *}$ & $.32^{* *}$ & $.70^{* *}$ & $.25^{* *}$ \\
\hline 7. Profesores (I) & & & & & & & 1.00 & $.46^{* *}$ & $.47 * *$ & $.46^{* *}$ & $.30 * *$ & $.77 * *$ & $.23^{* *}$ \\
\hline 8. Compañeros de clase (I) & & & & & & & & 1.00 & $.49 * *$ & $.42^{* *}$ & $.36^{* *}$ & $.76^{* *}$ & $.21 * *$ \\
\hline 9. Amigos cercanos (I) & & & & & & & & & 1.00 & $.29 * *$ & $.33^{* *}$ & $.74 * *$ & $.22 * *$ \\
\hline 10. Personas de mi facultad (I) & & & & & & & & & & 1.00 & $.40^{* *}$ & $.68^{* *}$ & $.20^{* *}$ \\
\hline 11. Apoyo social (F) & & & & & & & & & & & 1.00 & $.47 * *$ & $.49 * *$ \\
\hline 12. Apoyo social (I) & & & & & & & & & & & & 1.00 & 30** \\
\hline 13. Soporte Académico & & & & & & & & & & & & & 1.00 \\
\hline
\end{tabular}

Nota: $\mathrm{F}=$ Frecuencia, $\mathrm{I}=$ Importancia.

$* * p \leq .01 . * p \leq .05$. 
del factor personas de mi escuela por personas de mi facultad, se provocó cierto sesgo, debido a que los participantes pensaban que se preguntaba nuevamente por el soporte brindado por los profesores y compañeros de clase o por la figura no docente de la universidad (personal encargado de las cuestiones administrativas). Considerando estas dificultades se sugiere quitar dicho factor para estudios posteriores.

Por otro lado, son pocos los trabajos en la literatura que indagan las diferencias entre ambos sexos según la frecuencia e importancia de los soportes (Demaray \& Malecki, 2003a). Superando dicha dificultad, en este trabajo se observó que las mujeres presentaron mayores niveles de percepción de soporte social, según frecuencia e importancia, en las fuentes de padres y amigos cercanos. Estos resultados concuerdan parcialmente con lo reportado por algunos estudios previos, los cuales destacan mayor atención de las mujeres respecto a sus padres y compañeros de clase (Demaray \& Malecki 2002b; Malecki \& Demaray, 2003; Rueger et al., 2008). Por otro lado, las mujeres le atribuyeron mayor importancia a la percepción de soportes sociales; es probable, como indicaron Eschenbeck, Kohlmann y Lohaus (2007), que ellas destinen mayor cantidad de recursos para la generación de vínculos y esto se traduciría como una estrategia de afrontamiento ante diferentes situaciones.

En acuerdo a lo sostenido por varios estudios (Miranda et al., 2006; Fuligni et al., 1999; Crockett et al., 2007) y según lo estipulado en este trabajo, se observó que los estudiantes que no pertenecen a la ciudad sede de la universidad presentaron una mayor frecuencia en la percepción de los soportes de padres, profesores y personas de la facultad. Estos resultados indicarían que los estudiantes, al alejarse de su entorno de origen (familia y grupo de pares), tienden a establecer vínculos de soporte más sólidos con las fuentes previamente mencionadas. Sin embargo, solo los estudiantes de otras provincias y los que radican en la ciudad de Córdoba, a diferencia de los estudiantes del interior, fueron los que atribuyeron una mayor importancia al soporte brindado por los compañeros de clase. Una posible explicación podría deberse a que los estudiantes que provienen del interior podrían viajar con más frecuencia a sus hogares, debido a que el gasto económico es menor respecto a los estudiantes de otras provincias, y así mantener el contacto con sus pares.

Por otro lado, la escala de Soporte Académico evidenció resultados satisfactorios tanto en el estudio de validez de estructura interna y confiabilidad. Por su parte, el estudio de diferencia según la procedencia indicó que los estudiantes del interior de Córdoba presentaron un mayor nivel de percepción de soporte académico a diferencia de los estudiantes de dicha ciudad. A pesar que este resultado, parecería contradecir lo reportado previamente con el CASSS, donde los estudiantes de otras provincias son los que más perciben el soporte social, se debe considerar que la presente escala no diferencia la fuente que brinda el apoyo percibido a los estudiantes. En la última fase del estudio, si bien se encontró evidencia de validez convergente entre el CASSS y la escala de Soporte Académico, la magnitud de la asociación no fue la esperada, encontrado relaciones moderadas entre ambos constructos.

Respecto a las limitaciones del trabajo, se destaca la muestra de los participantes. La misma estuvo compuesta por un gran porcentaje de estudiantes que se encontraban realizando el curso de ingreso a la facultad. Esta desproporción podría haber distorsionado la percepción que poseen los estudiantes respecto a sus profesores, y compañeros de clase, debido a que la instancia de curso de ingreso implica un contacto con un entorno académico totalmente nuevo para el estudiante donde debe asimilar gran cantidad de información en poco tiempo con el objetivo de ingresar a la carrera. En adición, la mayor parte de los participantes de este estudio eran de sexo femenino. Por otro lado, los resultados expuestos en este trabajo respecto a la sub-escala personas de la facultad deben ser interpretados con precaución. Como se mencionó previamente, este factor presentó confusiones y dificultades por parte de los participantes, por tanto, se recomienda que futuros trabajos consideren una estructura factorial compuesta por los cuatro factores 
restantes. Por tanto, atendiendo a estas dificultades se sugieren nuevos estudios que contemplen las limitaciones aquí presentes e indaguen si la percepción de soporte social varía entre hombres y mujeres conforme los estudiantes progresan en sus carreras de grado e incluso si difieren en los niveles primario, secundario y universitario (Demaray \& Malecki, 2003b; Schraedley, Gotlib, \& Hayward, 1999).

Finalmente, como sugieren Rueger et al. (2010) respecto que el apoyo social podría operar de manera diferencial en cada cultura, particularmente en la población Hispana, este trabajo permitió observar cómo dicho constructo se desarrolla en una población de estudiantes universitarios de la ciudad de Córdoba (Argentina). La información aquí presentada puede ser utilizada para diseñar estrategias destinadas a estudiantes que presenten dificultades en su adaptación al contexto universitario, fortaleciendo los mensajes de apoyo de aquellas fuentes que los estudiantes consideren importantes. Como se destacó inicialmente, el fenómeno de deserción en las universidades públicas argentinas alcanza cifras preocupantes. En futuras investigaciones se debería explorar cómo estas variables ambientales, como el apoyo social y la percepción de ámbito universitario en conjunto con factores cognitivos, contribuyen a explicar la deserción académica. De este modo, la universidad podría evaluar el soporte percibido por los estudiantes y generar intervenciones que permitan fortalecer los espacios de interacción entre estudiantes, docentes y pares.

\section{Referencias}

Bologna, E. (2010). Estadística para Psicología y Educación. Córdoba: Ed. Brujas.

Brown, T. A. (2015). Confirmatory factor analysis for applied research. New York: Guilford Publications.

Cobb, S. (1976). Social support as a moderator of life stress. Psychosomatic medicine, 38(5), 300-314.

Cohen, J. (1988). Statistical power analysis for the behavioral sciences ( $2^{\mathrm{a}}$ ed.) Hillsdale, NJ: Erlbaum.

Cook, T. D., Herman, M. R., Phillips, M., \& Settersten, R. A. (2002). Some ways in which neighborhoods, nuclear families, friendship groups, and schools jointly affect changes in early adolescent development. Child Development, 73, 1283-1309.

Crockett, L. J., Iturbide, M. I., Torres Stone, R. A., McGinley, M., Raffaelli, M., \& Carlo, G. (2007). Acculturative stress, social support, and coping: Relations to psychological adjustment among Mexican American college students. Cultural Diversity and Ethnic Minority Psychology, 13(4), 347.

Demaray, M. K., \& Malecki, C. K. (2002a). Critical levels of perceived social support associated with student adjustment. School Psychology Quarterly, 17(3), 213-241. doi:10.1521/scpq.17.3.213.20883

Demaray, M. K., \& Malecki, C. K. (2002b). The relationship between perceived social support and maladjustment for students at risk. Psychology in the Schools, 39(3), 305-316.

Demaray, M. K., \& Malecki, C. K. (2003a). Importance ratings of socially supportive behaviors by children and adolescents. School Psychology Review, 32(1), 108-131.

Demaray, M. K., \& Malecki, C. K. (2003b). Perceptions of the frequency and importance of social support by students classified as victims, bullies, and bully/ victims in an urban middle school. School Psychology Review, 32(3), 471-490.

Domitrovich, C. E., \& Bierman, K. L. (2001). Parenting practices and child social adjustment: Multiple pathways of influence. Merrill-Palmer Quarterly, 47(2), 235-263.

Elias, M. J., \& Haynes, N. M. (2008). Social competence, social support, and academic achievement in minority, low-income, urban elementary school children. School Psychology Quarterly, 23(4), 474-495. doi:10.1037/1045-3830.23.4.474.

Eschenbeck, H., Kohlmann, C. W., \& Lohaus, A. (2007). Gender differences in coping strategies in children and adolescents. Journal of Individual Differences, 28, 18-26. doi:10.1027/1614- 0001.28.1.18. 
Ezeofor, I., \& Lent, R. W. (2014). Social cognitive and self-construal predictors of well-being among African college students in the US. Journal of Vocational Behavior, 85(3), 413-421. doi:10.1016/j. jvb.2014.09.003 0001-8791.

Flora, D. B., \& Curran, P. J. (2004). An empirical evaluation of alternative methods of estimation for confirmatory factor analysis with ordinal data. Psychological methods, 9(4), 466-491. doi:10.1037/1082989X.9.4.466.

Fuligni, A.J., Tseng, V., \& Lam, M. (1999). Attitudes toward family obligations among American adolescents with Asian, Latin American, and European backgrounds. Child development, 70(4), 1030-1044.

George, D., \& Mallery, P. (2011). IBM SPSS Statistics 21 step by step: $A$ simple guide and reference (13th ed.). Boston: Pearson Education.

Gresham, F. M., \& Elliott, S. N. (1990). Social skills rating system: Manual. United States:American Guidance Service.

Gresham, F. M., Elliott, S. N., \& Evans-Fernandez, S. E. (1993). Student Self-Concept Scale: Manual. United States:American Guidance Service.

Hair, J., Anderson, R., Tatham, R., \& Black, W. (1999). Análisis Multivariante (4 edición). España: Prentice Hall.

House, J. S. (1981). Work stress and social support. Reading, MA: Addison-Wesley Publishing Co.

Lent, R. W., Brown, S. D., \& Hackett, G. (2000). Contextual supports and barriers to career choice: A social cognitive analysis. Journal of counseling psychology, 47(1), 36.

Lent, R. W., Singley, D., Sheu, H. B., Schmidt, J. A., \& Schmidt, L. C. (2007). Relation of Social-Cognitive Factors to Academic Satisfaction in Engineering Students. Journal of Career Assessment, 15 (1), 87-97.

Lent, R.W., Brown, S. D., Sheu, L. B., Gloster, C. S., Wilkins, G., Schmidt, L. C., Lyons, H., \& Treistman, D. (2005). Social cognitive predictors of academic interests and goals in engineering: Utility for women and students at historically Black Universities. Journal of Counseling Psychology, 52, 84-92.
Little, R. J. (1988). A test of missing completely at random for multivariate data with missing values. Journal of the American Statistical Association, 83(404), 1198-1202.

Malecki, C. K., \& Demaray, M. K. (2002). Measuring perceived social support: Development of the child and adolescent social support scale (CASSS). Psychology in the Schools, 39, 1-18. doi: 10.1002/pits.10004

Malecki, C. K., \& Demaray, M. K. (2003). What Type of Support Do They Need? Investigating Student Adjustment as Related to Emotional, Informational, Appraisal, and Instrumental Support. School Psychology Quarterly, 18(3), 231-252. doi:10.1521/ scpq.18.3.231.22576

Malecki, C. K., Demaray, M. K., \& Elliott, S. N. (2003). A working manual on the development of the child and adolescent social support scale (2000). Unpublished manuscript. Northern Illinois University.

Mimura, C., \& Griffiths, P. (2008). A Japanese version of the Perceived Stress Scale: cross-cultural translation and equivalence assessment. $B M C$ psychiatry, 8(1), 85. doi: 10.1186/1471-244X-8-85

Miranda, A. O., Bilot, J. M., Peluso, P. R., Berman, K., \& Van Meek, L. G. (2006). Latino families: The relevance of the connection among acculturation, family dynamics, and health for family counseling research and practice. The Family Journal, 14(3), 268-273.

Nunnally, J. C. (1978). Psychometric theory (2nd ed.). New York: McGraw-Hill.

Reddy, R., Rhodes, J. E., \& Mulhall, P. (2003). The influence of teacher support on student adjustment in the middle school years: A latent growth curve study. Development and psychopathology, 15(1), 119-138.

Restubog, S. L. D., Florentino, A. R., \& Garcia, P. R. J. M. (2010). The mediating roles of career selfefficacy and career decidedness in the relationship between contextual support and persistence. Journal of vocational behavior, 77(2), 186-195.

Reynolds, C. R., \& Kamphaus, R. W. (1998). BASC: Behavior assessment system for children: Manual. American Guidance Service. 
Rueger, S. Y., Malecki, C. K., \& Demaray, M. K. (2008). Gender differences in the relationship between perceived social support and student adjustment during early adolescence. School Psychology Quarterly, 23(4), 496-514. doi:10.1037/1045-3830.23.4.496

Rueger, S. Y., Malecki, C. K., \& Demaray, M. K. (2010). Relationship between multiple sources of perceived social support and psychological and academic adjustment in early adolescence: Comparisons across gender. Journal of Youth and Adolescence, 39(1), 47-61.

Schafer, J. L. (1999). Multiple imputation: A primer. Statistical Methods in Medical Research, 8, 3-15.

Schraedley, P. K., Gotlib, I. H., \& Hayward, C. (1999). Gender differences in correlates of depressive symptoms in adolescents. Journal of Adolescent Health, 25, 98-108.

Schultheiss, D. E. P., Palma, T., Predragovich, K., \& Glasscock, J. (2002). Relational influences on career paths: Siblings in context. Journal of Counseling Psychology, 49, 302-330.

Secretaría de Políticas Universitarias (2013). Anuario de Estadísticas Universitarias. Argentina: Departamento de Información Universitaria. Ministerio de Educación de la Nación.

Skinner, E., Furrer, C., Marchand, G., \& Kindermann, T. (2008). Engagement and disaffection in the classroom: Part of a larger motivational dynamic? Journal of Educational Psychology, 100(4), 765-781.

Smith, M. C., Plant, M., Carney, R. N., Stein Arnold, C., Jackson, A., Johonson, L. S., Lange, H., Shane Mathis, F. \& Smith, T. J. (2003). Productivity of educational psychologists in educational psychology journals, 1997-2001. Contemporary Educational Psychology, 28, 422-430.

Tabachnick, B. G., \& Fidell, L. S. (2011). Using Multivariate Statistics (6th ed.). Needham Heights: Pearson.

Tardy, C. H. (1985). Social support measurement. American Journal of Community Psychology, 13(2), 187-202.

Verger, P., Combes, J. B., Kovess-Masfety, V., Choquet, M., Guagliardo, V., Rouillon, F., \& Peretti-Wattel, P. (2009). Psychological distress in first year university students: socioeconomic and academic stressors, mastery and social support in young men and women. Social psychiatry and psychiatric epidemiology, 44(8), 643-650.

Wettersten, K. B., Guilmino, A., Herrick, C. G., Hunter, P. J., King, G. Y., \& Jagow, D. (2005). Predicting educational and vocational attitudes among rural high school students. Journal of Counseling Psychology, 52, 658-663.

Yu, C. Y., \& Muthen, B. (2002, April). Evaluation of model fit indices for latent variable models with categorical and continuous outcomes. New Orleans, LA: Annual meeting of the American Educational Research Association. 